\title{
Quasi- and inelastic neutron scattering to investigate the molecular dynamics of discotic molecules in the bulk
}

\author{
Christina Krause $^{1}$, Reiner Zorn ${ }^{2}$, Bernhard Frick ${ }^{3}$ and Andreas Schönhals ${ }^{1, \text { a }}$ \\ ${ }^{1}$ BAM Federal Institute for Materials Research and Testing, Unter den Eichen 87, 12205 Berlin, Germany \\ ${ }^{2}$ Jülich Centre for Neutron Science, 52425 Jülich, Germany \\ ${ }^{3}$ Institut Laue-Langevin, 71, Avenue des Martyrs, 38000 Grenoble, France
}

\begin{abstract}
In- and quasielastic neutron scattering is employed to investigate both the vibrational density of states and the molecular dynamics of two homologous discotic liquid crystals (DLC) with different length of the alkyl side chain based on a triphenylene derivate. For both compounds characteristic low frequency excess contributions to the vibrational density of states are found. Therefore it is concluded that these liquid crystals show a glass-like behaviour. Elastic scans further show that in these materials a rich molecular dynamics takes place.
\end{abstract}

\section{Introduction}

Discotic Liquid Crystals (DLCs) are self-assembled materials. The molecules consist of a flat and rigid aromatic core substituted by aliphatic side chains [1-3]. Self-assembly is driven by noncovalent intermolecular interactions of the cores and the nonfavoral interactions of the cores and the side chains. At low temperatures DLCs exhibit a plastic crystalline phase. This phase can be followed by a hexagonal columnar liquid crystalline phase where the disc-shaped molecules organize into columns that further assemble into two-dimensional hexagonal arrays. The alkyl chains fill the intercolumnar space giving so rise to a nanophase separation. Besides hexagonal also nematic structures can appear [3].

Here the VDOS and the molecular mobility of two DLCs based on triphenylene are studied by neutron scattering. Besides of the technological importance of these materials, from a fundamental point of view it is interesting to study these complex systems in which order and mobility compete. This especially concerns the question whether features of glassy dynamics or glasses like characteristic excess contributions to the VDOS (Boson Peak, BP) are observed or not. For one of the studied materials (HAT6) neutron scattering data are already available in the literature where in addition to the protonated also the deuterated compound is considered. Moreover, the experimental data are compared to molecular simulations. For details see references [4-6] and work cited there. Employing inelastic neutron scattering phonons, electrons and charge transport have been considered (see for instance [7] and references cited there). Here special attention is paid to BP and the molecular mobility in the plastic crystalline and

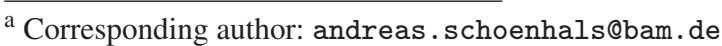

liquid crystalline phase covering also a larger temperature range.

\section{Experimental}

\subsection{Materials}

Two DLCs of a homologues series based on Hexakis(nalkyloxy)triphenylene (HATn) with $\mathrm{n}=5$ and $\mathrm{n}=6$ are used as model systems (chemical structure - inset of Fig. 1). The materials were purchased by Synthon Chemicals (Bitterfeld, Germany) and used as received. The thermal behavior was investigated by DSC (see Fig. 1 as example for HAT6). Both samples show a plastic crystalline phase at low temperatures, a hexagonal ordered liquid crystalline phase at intermediate and a isotropic phase with some remaining local order at high temperatures [8]. The phase transition temperatures are given in Table 1.

\subsection{Methods}

The neutron scattering experiments are carried out at the Institut Laue-Langevin (Grenoble, France). The VDOS was measured using the Time-of-Flight spectrometer IN6 at $80 \mathrm{~K}$ with an incident wave length of $5.12 \AA$ resulting in a resolution of $100 \mu \mathrm{eV}$ (full width at half maximum). The program INX [9] was used to correct the data for background and adsorption. The VDOS was measured at $80 \mathrm{~K}$. Figure 2 gives spectra as measured by IN6 at $80 \mathrm{~K}$ normalized to the height of the elastic peak. In addition a measurement at $2 \mathrm{~K}$ is also given serving as resolution $R(\omega)$ of the instrument.

The standard expression for the one-phonon scattering function [11] was employed to separate the low energy vibrations from the resolution of the instrument. Because of the fact that compared to $R(\omega)$ the $\operatorname{VDOS} g(\omega)$ is broad

This is an Open Access article distributed under the terms of the Creative Commons Attribution License 4.0, which permits unrestricted use, distribution, and reproduction in any medium, provided the original work is properly cited. 


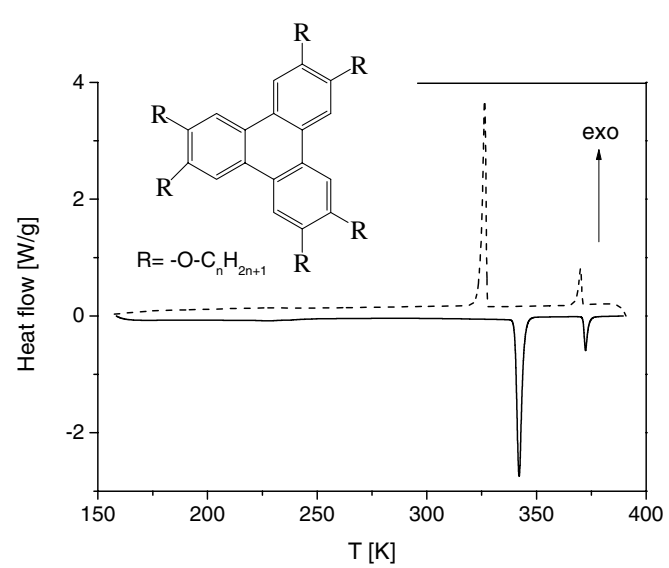

Figure 1. DSC thermogram for HAT6 during cooling (dashed line) and heating (solid line) at a rate of $10 \mathrm{~K} / \mathrm{min}$. Inset: chemical structure of HATn.

Table 1. Phase transition temperatures from the plastic crystalline to the hexagonal columnar phase $\mathrm{T}_{\text {cry,col }}$ and from the hexagonal columnar phase to the isotropic phase $\mathrm{T}_{\text {col,iso }}$ (second heating, heating rate of $10 \mathrm{Kmin}^{-1}$ ).

\begin{tabular}{|l|l|l|}
\hline Sample & $\mathrm{T}_{\text {cry, col }}[\mathrm{K}]$ & $\mathrm{T}_{\text {col, } \text { iso }}[\mathrm{K}]$ \\
\hline HAT5 & $341.7 \pm 1.5$ & $396.1 \pm 1.5$ \\
\hline HAT6 & $342.0 \pm 1.5$ & $372.3 \pm 1.5$ \\
\hline
\end{tabular}

convolution effects can be omitted and it approximately holds [12]

$$
\begin{aligned}
& S_{o b s}(q, \omega)=R(q, \omega) \otimes S(q, \omega) \\
& \approx \mathrm{e}^{-2 W(q)}\left(R(q, \omega)+\frac{\hbar q^{2}}{2 \bar{m}} \frac{g(\omega)}{-\omega} \times\left(1-\exp \left(\frac{\hbar \omega}{k_{B} T}\right)\right)^{-1}\right)
\end{aligned}
$$

where $\exp (-W(q))$ is the Debye-Waller factor, $\bar{m}$ the average mass of an atom, $k_{B}$ is the Boltzmann constant, and $q$ the scattering vector (momentum transfer). By measuring the scattering at two different temperatures allows the estimation of both the resolution and the vibrational density of states.

The neutron backscattering spectrometer IN10 was employed to obtain an overview about the molecular dynamics by elastic scans at a time scale of ca. $4 \mathrm{~ns}$ (resolution IN10 ca. $1 \mu \mathrm{eV}$ ). IN10 was used in standard configuration ('unpolished' Si-111) with a wave length of $6.271 \AA$. For the elastically scattered intensities the effective mean square displacement $<\mathrm{u}^{2}>_{\text {eff }}$ is extracted by a fractal model fit [13] which in case of a clear separation of elastic and inelastic scattering reduces to the usual Gaussian form

$$
I_{\mathrm{el}} / I_{0}=\exp \left[-q^{2}<u^{2}>_{\text {eff }} / 3\right] .
$$

$\left(\mathrm{I}_{\mathrm{el}}\right.$ and $\mathrm{I}_{0}$ are the elastically and totally scattered intensities) but is more reliable in the high $<\mathrm{u}^{2}>_{\text {eff }}$ value region. $\mathrm{I}_{0}$ was measured below $2 \mathrm{~K}$ with an increased statistics. The measurements were carried out with a heating rate of ca. $0.5 \mathrm{~K} / \mathrm{min}$. For both kinds of

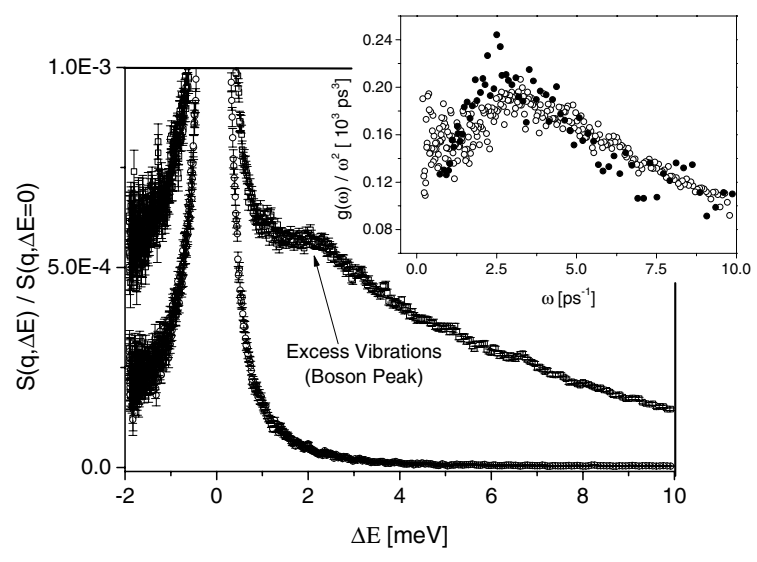

Figure 2. IN6 spectra of HAT6 normalized to the height of the elastic peak: squares $-\mathrm{T}=80 \mathrm{~K}$; circles $-\mathrm{T}=2 \mathrm{~K}$ representing the instrumental resolution. The data were averaged over the detector range $54 \ldots 108^{\circ}$ which is the range from which the VDOS was evaluated. The inset gives $\mathrm{g}(\omega) / \omega^{2}$ of HAT5 (empty circles) and of "amorphous" polyethylene (PE) (filled circles) given by Kanaya et al. [10]. The data for PE are scaled to collapse at the high frequency side with the HAT5 data.

measurements the scattering due to the empty can was subtracted.

\section{Results}

The materials under study consist of hydrogen $(\mathrm{H})$, carbon (C) and oxygen (O). Because of the fact that hydrogen is a strong incoherent scatterer and the coherent scattering cross sections are much smaller for all nuclei mainly the scattering due to the hydrogen nuclei are monitored and as result of the H-distribution in the molecule, at least for long alkyl chains, mainly the hydrogen in the alkyl chains (see inset Fig. 1). Therefore the observed scattering or dynamics can be assigned predominantly to the intercolumnar space.

\subsection{Vibrational density of states}

The data measured at IN6 at $80 \mathrm{~K}$ show a weak peak around an energy transfer of ca. $2 \mathrm{meV}$. This peak is due to excess contributions to the VDOS which are characteristic glass-forming materials or systems with a given disorder (frequency range $\omega=0.2 \ldots 1 \mathrm{THz}$ ). The Debye model of sound waves predicts $\mathrm{g}(\omega) \sim \omega^{2}$ for the VDOS. In the reduced representation $\mathrm{g}(\omega) / \omega^{2}$ versus frequency a peak is observed for these materials, which is commonly called Boson Peak (BP) (see inset Fig. 2) [14].The Boson Peak is a universal but controversially discussed feature of glasses and other materials with a complete or partial disorder. Moreover the BP corresponds to the excess contributions commonly found for glasses in the specific heat and in the thermal conductivity at low temperatures. As a matter of fact many systems which show a BP also exhibit a glass transition characterized for instance by the glass transition temperature $\mathrm{T}_{\mathrm{g}}$.

The inset of Fig. 2 displays the VDOS normalized to $\omega^{2}$ for HAT5. A Boson Peak is observed for this discotic liquid crystal in the plastic crystalline state. As already discussed above, as a BP seems to be 


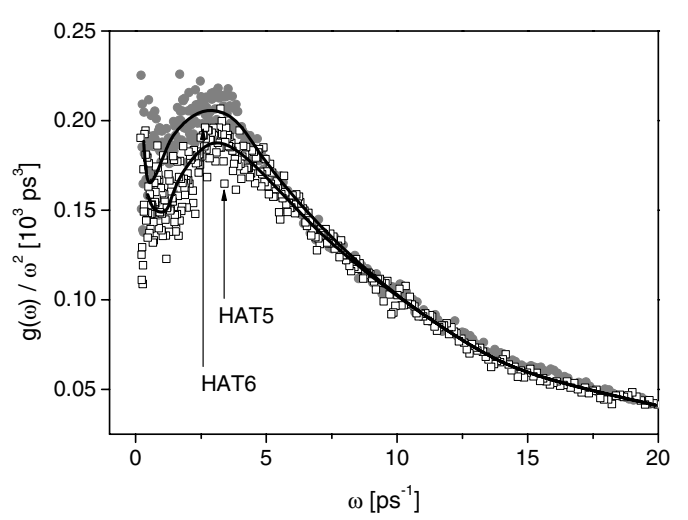

Figure 3. VDOS at $\mathrm{T}=80 \mathrm{~K}$ in the frequency range of the Boson Peak for HAT5 (open squares) and HAT6 (filled circles). The lines are guides for the eyes.

characteristic for a glassy behaviour one can conclude that this class of discotic liquid crystals in the plastic crystalline state resembles features of a glass. The observed scattering is mainly due the hydrogen atoms located in the intercolumnar regions. Moreover it was evidenced by X-ray investigations [8] that the alkyl chains have a more or less disordered structure. Therefore one can further conclude that these glassy features are related to the intercolumnar regions. The inset of Fig. 2 compares the Boson Peak for HAT5 with the vibrational density of states of "amorphous" polyethylene (PE) given by Kanaya et al. [10]. Here the term "amorphous" means that the VDOS was estimated for the amorphous regions of semicrystalline polyethylene constraint by the crystalline lamella [15]. It does not represent the VDOS for a purely (hypothetical) amorphous PE. With regard to both its position and its shape the Boson Peak measured for HAT5 corresponds to the VDOS estimated for PE. This indicates that the vibrational density of states measured for the methylene groups in the intercolumnar regions of HAT5 corresponds well to that for the $\mathrm{CH}_{2}$ groups in the constraint amorphous regions of semicrystalline polyethylene.

Figure 3 compares the Boson Peak of HAT5 and HAT6. The data for HAT6 are systematically higher than for HAT5 and the maximum of intensity is shifted to lower frequencies. Therefore the Boson Peak increases in intensity and shifts to lower frequencies going from $\mathrm{n}=5$ to $\mathrm{n}=6$ (see also reference [8]). Because of the fact that the Boson Peak is related to the elastic properties of the system of investigation $[16,17]$ this downshift of the maximum frequency of the Boson Peak corresponds to a softening of the material. Considering the morphology of the investigated DLCs the "soft" and flexible alkyl chains are arranged between the "hard" (stiff), ordered columns built up by the cores of the molecules. This can be regarded as a kind of a self-organized confinement superimposed by the columns to the alkyl chains. With increasing length of the side chain (HAT5 - > HAT6) the distance between the columns increases too. This might correspond to a partial release of the self-organized confinement imposed by the columns of the aromatic cores onto the methylene groups in the intercolumnar space. Within this picture one expects a shift of the Boson Peak to lower frequencies

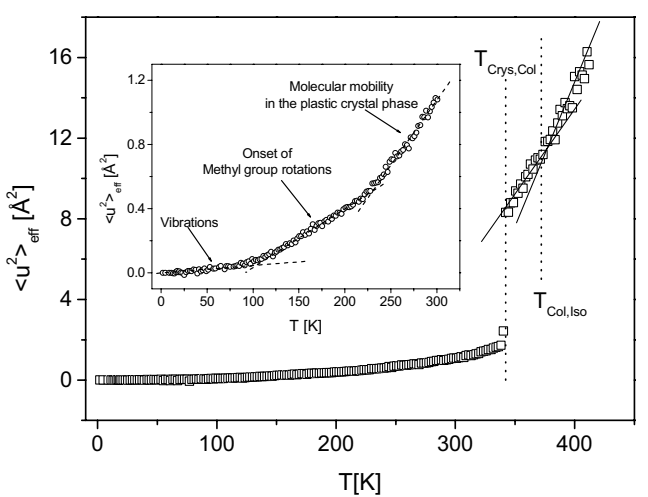

Figure 4. Temperature dependence of the effective mean square displacement $\left.<\mathrm{u}^{2}\right\rangle_{\text {eff }}$ for HAT6. The dotted lines correspond to the phase transition temperatures estimated by DSC. The inset enlarges the temperature dependence of $\left\langle\mathrm{u}^{2}\right\rangle_{\text {eff }}$ up to a temperature of $300 \mathrm{~K}$.

and an increase of its intensity. That is the opposite effect as observed when molecules are embedded in a tightened confinement. In that case the low frequency contributions of the Boson Peak decreasewith increasing confinement and therefore the maximum frequency of the Boson Peak shifts to higher frequencies (see for instance $[12,18,19]$ ). For a more detailed discussion see reference [8]. Moreover the influence of a hard nanometer confinement on the Boson Peak of HAT6 is considered in reference [20].

\subsection{Mean squared displacement}

Elastic scans measured at a backscattering spectrometer can be used to study molecular motion taking place at a larger length scale than vibrations. Figure 4 gives the temperature dependence of the effective mean square displacement $\left.<\mathrm{u}^{2}\right\rangle_{\text {eff }}$ for HAT6. First of all the both phase transitions of HAT6 are detected by changes of the temperature dependence of $<\mathrm{u}^{2}>$ eff. The phase transition temperatures estimated from $<\mathrm{u}^{2}>_{\text {eff }}(\mathrm{T})$ agree with the values measured by DSC.

The inset of Fig. 4 enlarges the temperature dependence of $\left\langle\mathrm{u}^{2}\right\rangle_{\text {eff }}$ for low temperatures. Different dynamic processes can be detected by changes of $<\mathrm{u}^{2}>_{\text {eff }}(\mathrm{T})$. At low temperatures the mean squared displacement is due to vibrations as discussed above. In the temperature range around $100 \mathrm{~K}$ the Methyl group rotation shows up as a step in $<\mathrm{u}^{2}>_{\text {eff }}$ [21]. At around $220 \mathrm{~K}$ motions at a larger scale within the plastic crystalline phase sets in.

Figure 5 compares $<\mathrm{u}^{2}>_{\text {eff }}(\mathrm{T})$ for HAT5 and HAT6 for low temperatures. For both DLCs the temperature dependence of $\left.<\mathrm{u}^{2}\right\rangle_{\text {eff }}$ is more or less similar. This is related to the fact that for these temperatures $<\mathrm{u}^{2}>_{\text {eff }}$ is much smaller than the core-core distance $\mathrm{d}$ $\left(\left(<\mathrm{u}^{2}>_{\text {eff }}\right)^{1 / 2} \ll \mathrm{d}\right.$, see inset of Fig. 5).

In the hexagonal ordered liquid crystalline phase the effective mean square displacement is larger for HAT6 than for HAT5 (see Fig. 6). This difference cannot be related to an Elastic Incoherent Structure factor do the localized motions (see Fig. 5). The mean square displacement is in the order of magnitude of the core-core distance of the columns $\left(\left(<\mathrm{u}^{2}>_{\mathrm{eff}}\right)^{1 / 2}<\mathrm{d}\right.$, see inset of 


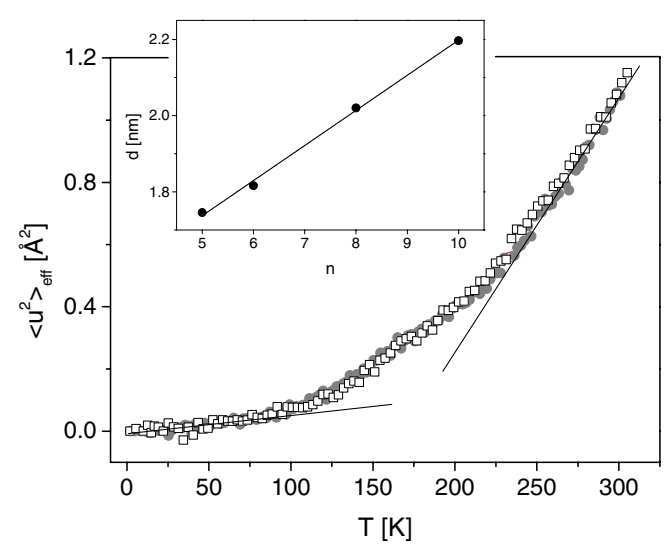

Figure 5. Temperature dependence of the effective mean square displacement $<\mathrm{u}^{2}>_{\text {eff }}$ for HAT5 (open squares) and HAT6 (filled circles) at lower temperatures in the plastic crystalline phase. The inset gives the core-core distance in the hexagonal ordered liquid crystalline phase of HATn versus the number of carbon atoms in the side chain according to [8].

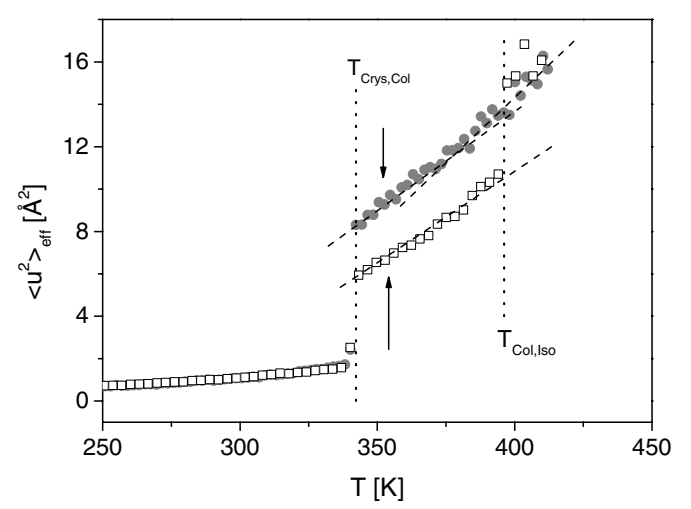

Figure 6. Temperature dependence of the effective mean square displacement $<\mathrm{u}^{2}>_{\text {eff }}$ for HAT5 (open squares) and HAT6 (filled circles) at higher temperatures in the hexagonal ordered liquid crystalline phase. The dotted lines correspond to the phase transition temperatures of HAT5 estimated by DSC. The dashed lines are guides to the eyes.

Fig. 5). As discussed for the density of states the columns of the aromatic cores impose a kind of self-organized confinement onto the diffusion of methylene groups in the intercolumnar space. This confinement will restrict the unlimited diffusion of the $\mathrm{CH}_{2}$ groups. Because the corecore distance is larger for HAT6 compared to HAT5 (see inset of Fig. 5) larger values for $<\mathrm{u}^{2}>_{\text {eff }}$ are observed for the DLC with longer side chains.

It is also interesting to note that at temperatures in the isotropic state the values for $<\mathrm{u}^{2}>_{\text {eff }}$ are similar. In this phase only a localized columnar structure is preserved [8]. This means the self-organized confinement imposed by the columns of the aromatic cores onto the diffusion methylene groups disappears and therefore comparable values of the effective mean square displacement are obtained.
The financial support by the German Science foundation (DFG SCHO 470/21-1) is gratefully acknowledged. The Institut Laue-Langevin is thanked for enabling the neutron scattering experiments.

\section{References}

[1] S. Chandrasekhar, B.K. Sadashiva, K. A Suresh, Pramana, 9, 471 (1977)

[2] J. Wu, W. Pisula, K. Müllen, Chem. Rev. 107, 718 (2007)

[3] S. Laschat, A. Baro, N. Steinke, F. Giesselmann, C. Hägele, G. Scalia, R. Judele, E. Kapatsina, S. Sauer. A. Schreivogel, T. Tosoni, Angew. Chem. 119, 4916 (2007)

[4] L. Haverkate, M. Zbiri, M. Johnson, B. Deme, F. Mulder, G. Kearley, J. Phys. Chem. B 115, 13809 (2011)

[5] F. Mulder, J. Stride, S. Picken, P. Kouwer, M. de Haas, L. Siebbeles, G. Kearley, J. Am. Chem. Soc. 125, 3860 (2003)

[6] G. Kearley, F. Mulder, J. Stride, S. Picken, P. Kouwer, Chemical Physics 292, 185 (2003)

[7] O. Kruglova, F. Mulder, A. Kotlewski, S. Picken, S. Parker, M. Johnson, G. Kearley, Chem. Phys. 330, 360 (2006)

[8] C. Krause, R. Zorn, F. Emmerling, J. Falkenhagen, B. Frick, P. Huber, A. Schönhals, Phys. Chem. Chem. Phys. 16, 7324 (2014)

[9] http://www.ill.eu/instruments-support/ computing-for-science/cs-software/allsoftware/tofhr/inx/

[10] T. Kanaya, K. Kaji, S. Ikeda, K. Inoue, Chemical Physics Letters, 150, 334 (1988)

[11] S. W. Lovesey, Theory of neutron scattering from condensed matter vol. 1 (Oxford University, New York, 1987)

[12] R. Zorn, L. Hartmann, B. Frick, D. Richter, F. Kremer, J. Non-Cryst. Solids, 307, 547 (2002)

[13] R. Zorn, Nucl. Instr. Meth. A 603, 439 (2009)

[14] R. Zallen, The physics of amorphous solids (Wiley, New York 1983)

[15] G. R. Strobl, The Physics of Polymers-Concepts for Understanding Their Structures and Behavior (Springer, Berlin 2007)

[16] M. T. Cicerone, C. L. Soles, Biophysical Journal, 86, 3836 (2004)

[17] W. Schirmacher, B. Schmid, C. Tomaras, G. Viliani, G. Baldi, G. Ruocco, T. Scopigno, physica status solidi (c), 5, 862 (2008)

[18] R. Zorn, B. Frick, L. Hartmann, F. Kremer, A. Schönhals, D. Richter, Physica B, 350, e1115 (2004)

[19] B. Frick et al. J. Non-Cryst. Solids 351, 2657 (2005)

[20] C. Krause, R. Zorn, B. Frick, A. Schönhals, Colloid Polym. Sci. 292, 1949 (2014)

[21] A. Schönhals, C. Schick, H. Huth, B. Frick, M. Mayorova, R. Zorn, J. Non-Cryst. Solids 353, 3853 (2007) 